\title{
Propuesta didáctica del poema Ricordi di Costarica de Massimo Fioravanti en una clase de italiano
}

\author{
AnNamaria Rimolo BariattI \\ Escuela de Lenguas Modernas \\ Universidad de Costa Rica
}

\begin{abstract}
Resumen
El objetivo del presente artículo es presentar una propuesta didáctica para la clase de italiano LE (como lengua extranjera) basada en la poesía de Massimo Fioravanti Bosi, un huelguista italiano en la Costa Rica del siglo XIX durante la construcción del ferrocarril al Atlántico, titulada Ricordi di Costarica y publicada en 1936. Para su elaboración nos fundamentamos en las nueve categorías de Hanauer (2001) para la construcción de significados. Por otro lado, es importante resaltar el hecho histórico de que el autor mismo comenta en dicha poesía su propia experiencia en Costa Rica así como que nunca haya sido publicada íntegramente en nuestro país. Fue escrita en la vejez del poeta campesino, luego de su regreso a Italia, en febrero de 1893.
\end{abstract}

Palabras claves: uso de la poesía en clase de italiano LE, David Ian Hanauer, construcción de significados, Ricordi di Costarica, Massimo Fioravanti Bosi, construcción del ferrocarril al Atlántico, huelga de los italianos en Costa Rica

\begin{abstract}
This article pretends to present a didactic proposal for the Italian class as a foreign language based on the poetry of Massimo Fioravanti Bosi, an Italian inmigrant and striker in the Costa Rica of the XIXth century, during the constrution of the railroad to the Atlantic coast, named Ricordi di Costarica that was published in 1936. In order to accomplish this proposal, we took into consideration the meaning construction process and its nine categories (Hanauer: 2001). It is important to enhance the historical facts that the author relates in this poem his life experience in Costa Rica and that it has never been published in its complete version in our country. It was written on February 1893, almost four years after the poet's return to Italy.
\end{abstract}

Key words: use of poetry in an Italian class as a foreign language, David Ian Hanauer, meaning construction process, Ricordi di Costarica, Massimo Fioravanti Bosi, railroad construction to the Atlantic Coast, Italians' strike in Costa Rica 


\section{Introducción}

$\mathrm{E}$ 1 objetivo de este trabajo es presentar una propuesta didáctica para estudiantes avanzados de Italiano LE (como lengua extranjera) que resalte la importancia del papel de la poesía en el aprendizaje. Para la elaboración de las actividades se echó mano de la sistematización de Hanauer (2001) en nueve categorías de los diferentes procesos mentales a la hora de comprender un poema.

El material (auténtico) utilizado para la presente propuesta didáctica es el poema Ricordi di Costarica del poeta campesino italiano Massimo Fioravanti Bosi. Forma parte de una compilación de poesías titulada Poesie (1936), donde el autor dedica un soneto y un poema a Costa Rica, país que le dio acogida como inmigrante trabajador en la construcción del ferrocarril al Atlántico a finales del siglo XIX. Hubo una edición anterior de las poesías de Massimo Fioravanti Bosi, bajo el título de Il canzoniere di un contadino, compiladas por algunos amigos y admiradores en 1904. Ricordi di Costarica fue escrito en febrero de 1893, casi cuatro años después del regreso del poeta campesino a Italia. Dada la importancia histórica de dicho poema y el hecho de que nunca haya sido publicado íntegramente en nuestro país -solamente un extracto de seis estrofas en Bariatti (2001: 87-88)-, se facilita el texto completo junto con su traducción libre en el apéndice.

Massimo Fioravanti Bosi nació en 1863 en Calto (provincia de Rovigo, en la parte sur oriental de la región del Véneto). Hijo de campesinos pobres, logró estudiar hasta tercer grado y luego tuvo que dedicarse al cultivo de la campiña para ayudar a sus padres. Seducido por el espejismo de una mejor suerte, junto con muchos otros compatriotas, llegó a Costa Rica en 1887 para trabajar en la construcción del ferrocarril al Atlántico. Se quedó hasta marzo de 1889, sufriendo las vicisitudes del emigrante abandonado a su suerte. Regresó pobre, trabajó de albañil en Roma, en tiempos de la "fiebre de la construcción" de fines de siglo XIX; esa experiencia fue negativa para él y decidió regresar a su pueblo natal, dedicado a la agricultura (Bariatti: 2001, 86).

En Poesie, Fioravanti Bosi utiliza una lengua italiana con inflexiones y arcaísmos típicos del siglo XIX, empero fácilmente inteligibles. En general, el poemario de Fioravanti Bosi relata experiencias pasadas y evoca nostalgia de los años juveniles. Acerca de su vivencia en Costa Rica como emigrante trabajador del ferrocarril, el autor dedica el soneto A Costarica y el corto poema Ricordi di Costarica. Ambas expresiones poéticas son relevantes por su contenido, que expresa un punto de encuentro concreto entre el mundo italiano y el mundo costarricense, algo prácticamente único en la poesía existente en ese momento. Además, es notable cómo Fioravanti Bosi logra revivir la experiencia inmanente y las dificultades encontradas en tierra ajena en el marco de una difícil situación laboral y social; importante es la percepción y la descripción de la Costa Rica de la década de 1880.

En el corto poema en que se basa nuestra propuesta didáctica para una clase de italiano LE, el poeta traduce con acento emotivo la dolorosa experiencia 
vivida en Costa Rica entre diciembre de 1887 y marzo de 1889. En el texto original, escrito mucho tiempo después del regreso a su patria, casi como una necesidad de liberar el espíritu lleno de la misma emoción de esos tiempos lejanos, se narran episodios de deslealtad y de explotación de los obreros italianos por parte del empresario ferrocarrilero.

\section{Las nueve categorías de Hanauer (2001)}

Hanauer (2001) en su estudio titulado "The Task of Poetry Reading and Second Language Learning" evalúa el papel de la poesía en la clase de lengua y su respectivo proceso de construcción de significados. Para ello, propone un experimento con veinte estudiantes avanzados de inglés LE según los lineamientos del enfoque cualitativo. Es decir, busca comprender cómo la poesía es leída y entendida. Divide la clase en parejas y les da la tarea de leer y comprender el significado de un poema en una canción popular. Graba la sesión. De los resultados, Hanauer (2001: 303-305) desarrolla una sistematización de los procesos mentales (coding system) involucrados en el proceso de construcción de significados en la interpretación de la poesía.

Dicha sistematización describe las diferentes respuestas de los estudiantes al leer y comprender el significado del poema. Son nueve categorías, a saber: noticing (frase evidenciadora), questioning (pregunta), interpretive hypothesis (hipótesis interpretativa), re-statement of an interpretive hypothesis (reelaboración de hipótesis interpretativa), counter statement of an interpretive hypothesis (frase opuesta a la hipótesis interpretativa), elaborative statement of an interpretive hypothesis (repetición de la hipótesis interpretativa), world knowledge (conocimiento global), integrating knowledge (conocimiento integral) y general statement (opinión).

La primera categoría (noticing) es definida como una frase en la cual el hablante dirige la atención hacia un aspecto específico del texto mediante diferentes maneras: señalando y/o citando líneas específicas, frases o palabras del poema; marcando repeticiones y/o diferencias entre líneas, frases o palabras específicas en el poema; evidenciando uso inusual gramatical dentro del poema; y señalando características literarias específicas dentro del mismo poema. La segunda categoría (questioning) consiste en preguntar algo relacionado con una sección o aspecto del poema, ya sea el significado específico de una oración, línea, frase o palabra, o su contenido.

Las siguientes categorías están relacionadas con la elaboración de hipótesis. La hipótesis interpretativa (interpretive hypothesis) constituye la tercera categoría y la más utilizada en el proceso de construcción de significados, donde el hablante interpreta con información no explícita en el poema para poder comprenderlo. La siguiente (re-statement of an interpretive hypothesis) se refiere a la confirmación por parte del hablante de una hipótesis interpretativa. Esto se hace mediante la repetición de lo interpretado anteriormente. Una frase opuesta a la hipótesis interpretativa (o su negación) es lo que constituye la quinta categoría 
(counter statement of an interpretive hypothesis). La sexta categoría (elaborative statement of an interpretive hypothesis) se basa también en la hipótesis interpretativa, pero agrega nuevas ideas o nueva información.

Cuando el hablante expresa una frase que evidencia su propio conocimiento general del mundo es lo que Hanauer identifica como la sétima categoría (world knowledge). Le sigue la categoría que asocia dos frases y, en consecuencia, produce una interpretación nueva del poema (integrating knowledge). Finalmente, expresar la propia opinión (general statement) constituye la última categoría involucrada en la construcción de significados en la comprensión de la poesía.

\section{Propuesta didáctica}

Ricordi di Costarica está dividido en cuatro secciones. En la primera sección de once estrofas, Fioravanti apela a sus compatriotas en Costa Rica y compañeros de sus desgracias en la construcción del ferrocarril al Atlántico. Los recuerda con dolor y tristeza. Pero con nostalgia también. Introduce al empresario del ferrocarril Minor Cooper Keith y su avaricia, quien trajo dos oleadas grandes de emigrantes italianos entre 1887 y 1888 para trabajar en la finalización de este proyecto, en el cual muchos encontraron la muerte (Bariatti, 2001: 71-108). En la segunda sección, compuesta también de once estrofas, el autor narra recuerdos específicos de Limón y de los peones, compañeros costarricenses en la construcción del ferrocarril. Describe el abandono del vicecónsul italiano en Costa Rica de sus propios compatriotas en los momentos de la huelga en el mercado de Cartago.

La tercera sección, de veintitrés estrofas, es la más larga y la más llena de optimismo. El poeta hace alusión a lo bueno vivido y visto en Costa Rica: sus montañas, sus selvas, su naturaleza y la eterna primavera. Relata también un poco de historia costarricense, de la fuerza del volcán Turrialba, de la primera destrucción de Cartago en 1841 (la segunda será en 1910). La cuarta sección está compuesta de veinte estrofas e inicia con el terremoto de 1888, la destrucción de Fraijanes y el dolor que conllevó para el país. En ellas Fioravanti continúa recordando la Costa Rica de fines del siglo XIX y sus costumbres: los toros, el guaro, la pelea de gallos y el buen trato de los costarricenses. Recuerda las vivencias como trabajador del ferrocarril en Quebrada Honda, donde estaba el campamento. Termina con su retorno -triste y abatido- a Italia en el vapor francés "Lesseps" que los transportó a él y a otros compatriotas desde Limón hasta Marsella.

La siguiente propuesta didáctica pone en evidencia el papel de la poesía en la clase de italiano LE al enfocarse en aquellos aspectos del poema Ricordi di Costarica que son lingüísticamente distintivos. Está diseñada para estudiantes avanzados. Siguiendo el diagrama de Lazar (2005: 127), está dividida en tres secciones: actividades prelectura (las dos primeras actividades), actividades durante la lectura (ocho actividades) y actividades poslectura (las tres últimas). A continuación, nuestra propuesta didáctica del poema Ricordi di Costarica de Massimo Fioravanti Bosi: 
Categoría

Hanauer

(2001)

World

knowledge

Elaborative statement of an interpretive hypothesis

\section{Integrating knowledge}

Noticing

Noticing

\section{Actividad en español}

Entren al blog titulado "Il blog per gli italiani in Costa Rica" (vigente desde 2009) dirigido a los italianos residentes en Costa Rica en la siguiente dirección electrónica: www.italiacostarica.com

Busquen el artículo titulado "In Costa Rica ceremonia per gli italiani caduti sul lavoro" escrito por Alfredo Ingegno, fechado el $01 \mathrm{de}$ octubre de 2011.

¿Qué saben del tema?

Piensen en la palabra 'inmigrante'. Escriban todas las imágenes que tengan con esta palabra.

Aquí está el poema Ricordi di Costarica de Massimo Fioravanti Bosi, un inmigrante italiano de fines del s.XIX, quien vino a Costa Rica a trabajar en la construcción del ferrocarril al Atlántico, traído por el empresario Minor Cooper Keith.

Lean el poema completo. Ahora comparen su visión de la palabra 'inmigrante' con la del poeta. ¿Comparten las mismas imágenes? ¿En qué sentido son diferentes?

Lean el poema y pongan atención al uso del lenguaje. No es italiano antiguo sino italiano del s.XIX. Es comprensible y fácil de entender. Busquen cinco diferencias entre el italiano utilizado por el autor Massimo Fioravanti Bosi y el italiano estándar.

Observen los préstamos del español costarricense como pezoz de plata (sección I, estrofa 8, línea 1) y ferrocarril (línea 3). ¿Cuáles otros préstamos encuentran en la sección II?

\section{Actividad en italiano}

Entrate sul blog intitolato "Il blog per gli italiani in Costa Rica" (creato nel 2009) per italiani residenti in Costa Rica nel seguente indirizzo elettronico: www.italiacostarica.com

Cercate l'articolo "In Costa Rica ceremonia per gli italiani caduti sul lavoro" scritto da Alfredo Ingegno, del 1 di ottobre del 2011.

Che ne sapete del tema?

Pensate alla parola 'immigrante'. Scrivete tutte le immagini che avete su questa parola.

Ecco il poema Ricordi di Costarica di Massimo Fioravanti Bosi, un migrante italiano del s.XIX, venuto in Costa Rica a lavorare nella costruzione della ferrovía, portato dall' imprenditore Minor Cooper Keith.

Leggete il poema completo. Fate un paragone sulla visione della parola 'immigrante' vostra con quella del poeta. Condividete le stesse immagini? In che senso sono diverse?

Leggete il poema y fate attenzione all'uso del linguaggio. Non è italiano antico ma un italiano del s.XIX. 亡̀ comprensibile e facile da capire. Cercate cinque differenze fra l'italiano del poeta e l'italiano standard.

Guardate i prestiti dallo spagnolo costarricense come pezoz de plata (parte I, strofa 8 , linea 1) e ferrocarril (linea 3). Che altri prestiti dallo spagnolo trovate nella sezione II del poema? 


\section{World \\ knowledge \\ Interpretive \\ hypothesis}

Questioning

Integrating

knowledge

Interpretive hypothesis

Re-statement of an interpretive hypothesis

Counter statement of an interpretive hypothesis

Noticing

Questioning

General statement
Contesten las siguientes preguntas: ¿En qué contexto histórico de Costa Rica se sitúa el autor?

¿Qué significado tiene la siguiente estrofa (sección I):

Ma sovra l'ossa mal sepolte e male

di martiri ingannati

sopra quell'ossa, nell'oblio perduto

van treni accelerati

¿Qué dice el autor de Limón?

¿Cómo la describe?

¿Qué cosa reclama Massimo Fioravanti al vicecónsul italiano en Costa Rica en ese período de 1887 a 1889? ¿Cómo se relaciona este pasaje de la sección II (últimas cuatro estrofas) con la primera huelga en Costa Rica?

En la sección II, la tercera y cuarta estrofas se refieren a los peones de la construcción del ferrocarril al Atlántico. ¿Cómo los describe?

¿Cómo este inmigrante italiano los describe en su frase:

e l'usanza di dar le scarpe ai morti non lavorate in vita?

En la sección II, estrofas 6 y 8 , el poeta relata su propia experiencia como trabajador en la construcción del ferrocarril al Atlántico. ¿Cómo fue?

¿Qué significa la frase senza rossor nel viso?

¿Qué significan las frases (sección III, estrofa 7) l'eterna primavera y l'eterna estate? ¿No se contradicen estas imágenes con la línea dove la febbre impera?

En la sección IV, el poeta habla de las costumbres en la Costa Rica del s.XIX. Enumere tres.

¿Cómo terminan las vivencias del poeta en Costa Rica?

¿Les gustó el poema? Si así fue, expliquen sus razones. Si no les gustó, ¿por qué no?
Rispondete alle seguente domande: In quale contesto storico del Costa Rica si trova il poeta?

Che significa la seguente strofa (parte I):

Ma sovra l'ossa mal sepolte e male di martiri ingannati sopra quell'ossa, nell'oblio perduto van treni accelerati

Che cosa vuol dire l'autore?

Come la descrive?

Di che cosa si lamenta Massimo Fioravanti del Vice-console italiano in Costa Rica dal 1887 al 1889?

Come si collega questa parte del poema (parte II, le ultime quattro strofe) con il primo sciopero in Costa Rica?

Nella parte II, la terza e quarta strofe parlano dei 'pioni' nella costruzione della ferrovía all' Atlantico. Come sono? Come questo immigrante italiano li descrive in questa frase:

e l'usanza di dar le scarpe ai morti non lavorate in vita?

Nella parte II, strofe 6 e 8 , il poeta parla della sua sperienza come lavoratore nella costruzione della ferrovía all'Atlantico. Come è stata questa esperienza? Che significa la frase senza rossor nel viso?

Che cosa significano le frasi (parte III, strofa 7) l'eterna primavera y l'eterna estate? Non si contraddiscono queste immagini con la linea dove la febbre impera?

Nella parte IV, il poeta parla delle abitudini nella Costa Rica del s.XIX. Enumeratene tre.

Come finisce la storia del poeta in Costa Rica?

Vi è piaciuto il poema? Perché sí o perché no? 


\begin{tabular}{|c|c|}
\hline $\begin{array}{l}\text { Re-statement } \\
\text { of an interpre- } \\
\text { tive hypothesis }\end{array}$ & $\begin{array}{l}\text { Formen grupos de tres. Escojan un } \\
\text { par de estrofas del poema y dra- } \\
\text { matícenlas. }\end{array}$ \\
\hline ive & Vean el corto titulado "Costa \\
\hline & $\begin{array}{l}\text { Rica" de un turista italiano en } \\
\text { nuestro país en la siguiente di- } \\
\text { rección } \\
\text { watch?v=EhBoutube.com/ } \\
\text { wwo }=\text { Pob1K1 de } 12.45^{\prime} \text {. }\end{array}$ \\
\hline & Hagan una lista de descripciones de \\
\hline & $\begin{array}{l}\text { Costa Rica según su autor Sergio de } \\
\text { Giacomo. }\end{array}$ \\
\hline ues & $\begin{array}{l}\text { aspectos de Costa Rica le lla- } \\
\text { a atención al turista italiano? }\end{array}$ \\
\hline ount & Comparen esas imágenes con la Cos- \\
\hline & . \\
\hline & encuentran? \\
\hline
\end{tabular}

Dividetevi in gruppi di tre. Scegliete un paio di strofe del poema e drammatizzatele.

Guardate il video titolato "Costa Rica" di un turista italiano nel nostro paese nel seguente indirizzo: www.youtube. com/watch?v $=$ EhBp7P8b1K1 di $12.45^{\circ}$.

Fate un listino delle descrizioni che Sergio de Giacomo fa del Costa Rica.

Che cosa attira l'attenzione del turista italiano?

Condividete queste immagini con la Costa Rica di Fioravanti nel poema. Che somiglianze e differenze trovate?

\section{Conclusiones}

La presente propuesta didáctica basada en el poema Ricordi di Costarica del huelguista italiano Massimo Fioravanti Bosi en el período de la construcción del ferrocarril al Atlántico de finales del siglo XIX le permite a los estudiantes avanzados de italiano LE (como lengua extranjera) la posibilidad de extender su comprensión de los potenciales usos y significados de la estructura lingüística dentro del poema. Además, les permite desarrollar conciencia cultural e histórica al observar la distancia entre el texto discursivo, el contenido del poema y el propio conocimiento de los mismos estudiantes. En conclusión, el poema permite a los estudiantes ampliar su conocimiento lingüístico y cultural de la lengua italiana, mediante la aplicación de las nueve categorías de Hanauer (2001) que componen el proceso de construcción de significados en una clase de lengua. El material auténtico utilizado en la presente propuesta didáctica es único por dos razones. La primera es su importancia histórica, puesto que fue escrito por un poeta campesino italiano, quien participó en la "huelga de los italianos" en Cartago a raíz de las malas condiciones laborales que privaban en la construcción del ferrocarril al Atlántico al finalizar el siglo XIX. La segunda razón es que no ha sido publicado en su totalidad sino hasta el día de hoy en el presente artículo.

\section{Apéndice}

A continuación la transcripción del poema Ricordi di Costarica de Massimo Fioravanti Bosi (1936: 89-105) con su respectiva traducción libre al español. 


\section{RICORDI}

Ad alcuni miei amici di Costarica (1)

\section{I}

Voi di Santo Domingo, amato Sante, Voi, caro Federico,

Voi dell'Hotel de Roma, o Sacripante, Voi, Calivá, mio amico.

Io Vi ricordo da molt'anni e v'amo, o americani amici,

e ai Vostri monti ancor che siate bramo viviate felici.

I nomi vostri allor che il mio dolore alleggeriste tanto

a lettere indelebili nel core

me li scrissi col pianto.

Quando raccolto nella mia tristezza e da nessun protetto

vi designaste, per la mia gramezza, la carità un diletto.

Quando fuggimmo dall'altura alpestre (2) i bassi accampamenti (3)

in cui natura tante cose ha destre

a preparar tormenti.

Già per quei monti in veste di fustagno or d'ire avrà finito

il magro imprenditor, ma dal guadagno or di seta vestito

pezoz de plata (4) intascherà a milioni

da $\mathrm{S}$. Josè risortoç

lungo il ferrocarril (5) nelle stazioni

e di Limone al porto:

di Costarica in seno or più non langue commercio affaticato:

quell'arteria del mar gli porta sangue e migliora il suo stato.

Ma sovra l'ossa mal sepolte e mute di martiri ingannati (6) sopra quell'ossa, nell'oblio perdute, van treni accelerati

ed ivi in ogni canto al fischio acuto, che manderà il vapore,

dal grigio spettro d'un pion (7) caduto risponderà un dolore.

\section{RECUERDOS}

A mis amigos de Costa Rica (1)

\section{I}

Vosotros de Santo Domingo, querido Sante, vosotros, querido Federico,

vosotros del Hotel Roma, oh Sacripante, vosotros, Calivá, amigo mío.

Yo os recuerdo desde hace muchos años y os amo,

oh amigos americanos, y os deseo que estando aún en vuestras montañas,

felices viváis.

Entonces vuestros nombres que mi dolor han mucho aliviado

con letras indelebles en mi corazón

los escribí en llanto.

Cuando recogido en mi tristeza

y de nadie protegido

habíais escogido, para mi miseria,

el placer de la caridad.

Cuando huímos de la altura (2)

los bajos campamentos (3)

donde la naturaleza tiene cosas capaces

de preparar tormentos.

Ya por esos montes en vestido de paño

habrá terminado de caminar

el flaco empresario; pero con las ganancias ahora vestido de seda.

Embolsará pesos de plata (4) por millones desde San José, reflorecido

a lo largo del ferrocarril (5) en las estaciones y de Limón al puerto.

En Costa Rica ya no languidece

un comercio estancado:

esa arteria de mar le trae sangre

y mejora su estado.

Pero sobre los huesos mal enterrados y mudos de los mártires engañados

sobre esos huesos, perdidos en el olvido, van trenes acelerados

y allí en cada silbato agudo

que enviará el vapor,

del gris espectro de un peón (7) caído

contestará el dolor. 
Se all'anglo imprenditor Limon dà gloria e alla vita dei porti

oh! Del ferrocarril taccia la storia

il numero dei morti!

\section{II}

Di piante ignudo alla silvestre costa entre lo spazio aperto

dove qualche piroscafo fa sosta, non di agrumi deserto,

mi ricorda Limon, caldo e malsano senza traccia di fede, che solo, accanto al mar, nel suo pantano a far da porto siede.

Allor di pochi negri era il soggiorno per cui nell'ermo loco passava gramo e senza vita il giorno come a nati per gioco.

I candidi, ricordo, inamidati

e lucidi calzoni

che coi lor piedi scalzi e logorati

indossano i pioni.

Ricordo i loro gabbanelli corti, los sombreros de pita (8)

e l'usanza di dar le scarpe ai morti non logorate in vita.

Né l'ore tristi del dolor mi scordo in cui la fame ardea

al focolar del mio digiuno ingordo

e furtivo piangea!

Pur di quei dì la carità rammento di S. Josè pietosa (9)

che della fame ci lenì il tormento come madre amorosa.

E ne l'abbandono, itali perduti, ci dispensaba il riso:

che mangiavamo per le vie seduti senza rossor nel viso.

Perchè quel Vice-Console, dottore, (10) della sua patria figli, ci lasciava soffrir tanto dolore, ci abbandono' ai perigli?

Forse il dottor nei torbidi momenti, tanto brutti, per noi, pensava intorno al mal de' suoi clienti o agli interessi suoi.
Si al empresario Limón da gloria y a la vida de los puertos ¡oh del ferrocarril calle la historia el número de muertos!

\section{II}

De plantas desnudo en la silvestre costa entre el espacio abierto

donde unos navíos hacen parada, no desierto de limoneros, me recuerda Limón, caliente y malsano sin trazos de fe, que solo, junto al mar, en su pantano como puerto se sostiene.

Entonces de pocos negros era la estadía por lo que en ese solitario lugar pasaba pobre y sin vida el día como los nacidos por juego.

Los blancos, recuerdo, almidonados y brillantes pantalones que con sus pies descalzos y desgastados se ponen los peones.

Recuerdo sus vestimentas cortas, los sombreros de pita (8)

y la costumbre de poner a los muertos zapatos nunca gastados en vida.

Ni de las horas tristes del dolor me olvido en que el hambre ardía

al fuego de mi gran ayuno

¡Y en secreto lloraba!

De esos días, la caridad recuerdo de una San José piadosa (9), que del hambre nos alivió el tormento como madre amorosa.

Y en el olvido de itálicos perdidos, nos proporcionaba el arroz: que comíamos por la calle, sentados, sin rubor en el rostro.

¿Por qué ese vicecónsul, doctor, (10)

a nosotros de su patria hijos

nos dejaba sufrir tanto dolor, nos dejó abandonados a los peligros?

Talvez el doctor en los turbios momentos, tan feos, para nosotros, pensaba en el mal de sus clientes o en los intereses suyos. 
Di quei giorni d'angoscia e di bisogno le rie torture lente come il quadro lontan di triste sogno ancora ho nella mente.

\section{III}

Nel cor pur mi resto' Santo Domingo, la vostra compagnia, in cui non fui più l'esule solingo $o$ il cane della via.

Che la rugiada e il sol dipingon d'oro ricordo le foreste di cui le piante son del dorso loro ancor la prima veste.

Sorge tra il verde la maestosa altura grande nel cheto oblio;

par che dal fondo della sua natura s'alzi a parlar di Dio.

Quelle leggiadre gigantesche selve che, pria del sol, l'aurora vergini saluta, senza le belve, voi le vedete ancora,

e son così da un'ordine guidate, esperimento piano,

del Fattor loro in grembo al dì create ad onorar la mano.

Ricordo i vostri deliziosi piani alle montagne in vetta che d'ingoiar minacciano i vulcani nell'ira maledetta.

Ricordo delle alture, ove abitate, l'eterna primavera

e al basso, verso il mar, l'eterna estate dove la febbre impera.

Di leggiadra natura, onor selvaggio, lungi alla colte aiuole

che l'aura lambe e col suo mite raggio bacia soltanto il sole;

dell'anima ricordo il vostro fiore, (11) di grato odor sì adorno,

che nel silenzio alpestre il suo colore cangia tre volte al giorno:

e poscia lento a dissecar si perde entre assoluto oblio curvo, de' prati fra l'eterno verde, sovra lo stel natio.
De esos días de angustia y de necesidad las malvadas torturas lentas como el cuadro lejano de un triste sueño aún tengo en mi mente.

\section{III}

En el corazón me quedó Santo Domingo, vuestra compañía, en la cual yo no fui más el exiliado solo o el perro de la calle.

Que el rocío y el sol pintan dorados recuerdo los bosques cuyos árboles tienen aún en su corteza la primera envoltura primaveral.

Surge entre el verde la majestuosa altura grande en el silencioso olvido; parece que desde el fondo de su natura se levante a hablar de Dios.

Esas livianas gigantescas selvas que, antes del sol, la aurora vírgenes saluda, sin las fieras, vosotros aún las veis, y son así por un orden guiadas, experimento suave, del Hacedor en el día creadas para honrar su mano.

Recuerdo vuestros deliciosos llanos en las cumbres de las montañas que de tragar amenazan los volcanes en la ira maldita.

Recuerdo de las alturas, donde habitáis, la eterna primavera y abajo, hacia el mar, el eterno verano donde la fiebre impera.

De armoniosa naturaleza, honor salvaje, a lo largo de cuidados céspedes que la brisa tantea y con su dulce rayo besa únicamente el sol;

del alma recuerdo vuestra flor (11) de agradable fragancia tan ataviada, que en el silencio alpestre su color cambia tres veces al día:

y luego lenta a secarse se pierde entre el absoluto olvido curva, entre el eterno verde de los prados, sobre el tallo nativo. 
Ricordo S. Josè fra i monti assiso quale di gioie un velo dove par che raccolgano il sorriso Arte, natura e cielo.

Dove la militar famiglia intera, quando il bisogna implora, di fanti scalzi, a paga giornaliera, la Capitale onora.

Ricordo ancor la placida Cartago (12) in cui Torialba (13) un giorno fè distruggendo la sua bella imago del terrore il soggiorno;

ed or sulle rovine edificata tanto bella s'asside dolcemente dai fiori profumata tra cui nel dì sorride.

Ricordo il vostro ciel di sotto al quale han d'aleggiar la cura

la notte al dí, e il dí alla notte uguale, che l'ordine misura.

Ricordo il vostro sol torrido e mite che d'april e d'agosto per due meriggi delle piante ardite non lascia all'ombre un posto.

Di raggi allor che al giorno il sol distende la fulgida criniera, come dall'alvear l'ape discende, nell'ora mattiniera,

uscir dal verde sen della foresta un dì vid'io l'indiano di cui non ancor domina la gesta nessun progresso umano.

Ei timidetto, in mezzo a noi passava nudo, guardando il suolo

e a mo' di cinghia alle vergone usava misero cencio solo.

D'esso natura ancor non si ridesta a risvegliar la mente, nè lo spinge a salir dalla foresta alla moderna gente...

Ma in esso ancor non pratico' il rancore il suo funesto assaggio, nè l'odio, come in noi, guasto' l'amore del suo pensier selvaggio.
Recuerdo San José situada entre los montes como de alegrías un velo donde parece que recogen la sonrisa arte, naturaleza y cielo.

Donde la militar familia entera, cuando la necesidad implora, de soldados descalzos, a sueldo diario la Capital honra.

Recuerdo aún la plácida Cartago (12) de la cual el Turrialba (13) un día hizo destruir su linda imagen del terror la estadía;

y ahora sobre las ruinas construida tan bella se asienta dulcemente por las flores perfumada entre las cuales en el día sonríe.

Recuerdo vuestro cielo bajo el cual deben soplar con cuidado de la noche al día, y del día a la noche igual, como el orden impone.

Recuerdo vuestro sol ardiente y suave que en abril y en agosto por dos mediodías a las plantas intrépidas no les deja a la sombra un lugar.

De los rayos que en el día el sol extiende su resplandeciente melena, como de la colmena la abeja desciende, en la hora matutina,

salir del verde pecho de la selva un día yo vi al indio del cual aún no domina la gesta ningún progreso humano.

Él timidillo, en medio nos pasaba desnudo, mirando al suelo y como cinturón en las vergüenzas usaba un mísero trapo solo.

Para él la naturaleza aún no se despierta a despejar la mente ni lo empuja a salir de la floresta a la moderna gente...

Pero en él aún no practicó el rencor su funesta experiencia, ni el odio, como en nosotros, descompuso el amor de su pensamiento salvaje. 
Calmo egli vive fra selvaggia quiete lungi ai contrasti umani, nè di grandezza calorosa sete lo spinge a tristi piani.

Ei le discordie nostre ancor non ode, i nostri mali ignora, e ad esso acuta invidia il cor non rode come il nostro divora.

\section{IV}

Ancor d'un templor (14) tanto violento, udir parmi il boato,

dalla terra venir come un lamento di tuono soffocato.

$\mathrm{E}$ a fare più terribile e più forte fra l'umano sgomento il terror dell'abisso e della morte, il sì grave momento:

come sinistra lampada che manda luce terrorizzante

e che senza pietà a piombar la spanda sulla terra ondulante:

dalla gran face del sinistro monte, che tanto irosa ardea, fumo esalava colle vampe in fronte che rosso il ciel facea.

E le campane poco in alto assise, tanto squassate e mosse mandavan note di lamenti intrise. E il suon parea che fosse il segno funeral dell'ingoiata famiglia d'un dottore di cui la moglie, sola e disperata, resto' viva al dolore.

Rammento il gioco barbaro dei tori, che ancor non so se usiate, l'aguaro (15), i galli in guerra, i vostri amori, le bibite gelate.

Ma più d'ogni costume e d'ogni loco, che bello vi circonda, e più d'ogni bellezza e d'ogni gioco, ricordo Quebrada Honda, (16)
Tranquilo él vive entre la salvaje quietud lejos de los contrastes humanos, ni de grandeza una calurosa sed lo empuja a funestos designios. Él los conflictos nuestros aún no oye y nuestros males ignora, y a él aguda envidia el corazón no roe como el nuestro devora.

\section{IV}

Aún de un temblor (14) tan violento, oír me parece el estruendo de la tierra venir como un lamento de trueno ahogado.

Y a hacer más terrible y más fuerte entre la humana desolación el terror del abismo y de la muerte, el tan grave momento:

como siniestra lámpara que envía luz terrorífica y que sin piedad al caer la esparce sobre la tierra ondulante:

desde la gran antorcha del siniestro monte, que tan iracunda ardía,

humo emanaba con bochornos en la frente que rojo el cielo volvía.

Y las campanas un poco encima colocadas, todas percudidas y movidas mandaban notas de quejas empapadas. Y el sonido parecía que fuera

la señal funeraria de la sepultada familia de un doctor del cual, la esposa, sola y desesperada quedó viva en el dolor.

Recuerdo el juego bárbaro de los toros, que aún no sé si lo usáis, el guaro (15), los gallos de pelea, vuestros amores, las bebidas heladas.

Pero más que cada costumbre y cada lugar bello que os circunda,

y más que cada belleza y cada juego, Quebrada Honda (16) recuerdo, 
in cui col dì nascente in un pensiero dolce cordial tributo, della famiglia coll'affetto intero mi giungeva il saluto:

e colla mente in un desìo confusa e l'agitato core

dove l'anima mia un dì s'è fusa in conforto e dolore.

E del gigante Palma (17) alla discesa dove commossi e muti

noi ci guardammo in viso alla sorpresa degli estremi saluti.

Di quel nostro distacco, amaro tanto, sempre ricordo il giorno

in ci accompagno' coi baci il pianto facendo al mar ritorno.

Io ero forte allor, nè m'opprimea del dì l'aspra fatica,

e fra le mie tristezze aver solea qualche speranza amica.

Ed ora stanco nella vita assiso, ridotto inutil pondo,

che molesta l'età, or che un sorriso per me non ha più il mondo,

assieme ai vostri generosi cuori,

ignari degli inganni, assieme ai vostri monti e ai vostri fiori ricordo i miei vent'anni.

Così come il pensier su bianca tenda Mi vi dipinge or ora, anzi che il sol per me più non risplenda vorrei vedervi ancora.

Ma fra la speme ormai non m'è più sconta la verità segnata, ed al fosco orizzonte che tramonta vedo la mia giornata.

E d'affrontar d'un mare così vasto il periglioso viaggio or, dell'età agli sgoccioli rimasto, non mi sento il coraggio.

Perchè dal dì che mossi il passo gramo verso straniera via ho potuto capir quanto io l'amo la cara Patria mia: donde al nacer el día en un pensamiento dulce cordial tributo, de la familia con todo el afecto me llegaba el saludo:

y con la mente en un deseo confusa y el agitado corazón donde el alma mía un día se derritió en consuelo y dolor.

Y del alto monte Palma (17) a la bajada donde emocionados y mudos nos miramos a la cara con la sorpresa de los extremos saludos.

De ese nuestro despegue, tan amargo, siempre recuerdo el día en que nos acompañó con besos el llanto haciendo al mar el retorno.

Yo era fuerte entonces, ni me oprimía del día la áspera fatiga, y entre mis tristezas tener solía alguna esperanza amiga.

Y ahora cansado en la vida colgado, reducido a inútil pienso, qué molesta la edad, ahora que una sonrisa para mí ya no tiene más el mundo,

junto con vuestros generosos corazones, ignorantes de los engaños, junto con vuestros montes y vuestras flores recuerdo mis veinte años.

Así como el pensamiento sobre blanca pantalla os pinta ahora, antes de que el sol para mí ya no resplandezca quisiera veros de nuevo.

Pero en la esperanza ya no me es concedida la verdad señalada, y en el sombrío horizonte que decae veo mi jornada.

$Y$ para enfrentar un mar tan vasto el peligroso viaje ahora, de la edad lo último que ha quedado, no me siento con valor.

Porque desde el día en que moví el paso difícil hacia el destino extranjero he logrado entender cuánto yo amo la querida Patria mía: 
e finita col dì l'acerba guerra godere assai mi piace nel dolce seno della patria terra il sonno della pace. y terminada con el día la áspera guerra

disfrutar bastante me gusta

en el dulce refugio de la patria tierra

el sueño de la paz.

\section{Notas de Massimo Fiorvanti Bosi a lo largo del poema Ricordi di Costarica (ibid.).}

1. A Costarica vissi dal dicembre 1887 al marzo 1889, emigrato in quella piccola Repubblica per lavorare nella costruzione della Ferrovia, con un'Impresa Inglese.

En Costa Rica viví desde diciembre 1887 hasta marzo 1889 como inmigrante inmigrante para trabajar en la construcción del ferrocarril al Atlántico con una empresa inglesa (nota de traducción: en realidad es del empresario estadounidense Minor Cooper Keith).

2. Avendo mancato l'Impresa a' suoi patti e avendo preteso di mandare i lavoratori italiani in posizioni mal sane, abbiamo scioperato andando tutti a Cartago, piccola ma bella cittadina posta fra porto Limone e la capitale S. Josè.

Cuando la Empresa incumplió con lo pactado y decidió enviar a los trabajadores italianos a lugares insalubres, hicimos huelga y fuimos todos a Cartago, una ciudad pequeña pero bella situada entre Puerto Limón y la capital de San José.

3. Capanne di canna costruite in base gole delle montagne.

Cabañas de caña construidas al pie de las montañas.

4. Scudi di metallo.

Monedas de plata.

5. Strada ferrata.

Vía férrea.

6. Lavoratori morti lungo la línea che si costruiva.

Trabajadores muertos a lo largo de la línea del ferrocarril que se construía.

7. Uomo operaio.

Trabajador o peón.

8. Cappelli di paglia a larghe tese.

Sombreros de paja de solapa ancha.

9. Capitale di Costarica.

Capital de Costa Rica.

10. Un uomo piccolo, toscano, che fu nostro medico di bordo e poscia nominato

Vice-Console Italiano a Costarica.

Hombre pequeño, toscano, que fue nuestro médico de a bordo, luego fue

nombrado Vicecónsul de Italia en Costa Rica.

11. Un fiore che gli abitanti di quei luoghi chiamavano "dell' anima".

Una flor que los habitantes de aquellos lugares llamaban "del alma".

12. Piccola città a sud-est di S. Josè.

Pequeña ciudad al sureste de San José.

13. Vulcano che la distrusse.

Volcán que la destruyó. Nota de traducción: la primera destrucción de Cartago se dio el 2 de setiembre de 1841 y la segunda en 1910 (González Víquez, 1994: 233).

14. Terremoto.

Terremoto. Nota de traducción: el 30 de diciembre de 1888 se dio un terremoto con gran destrucción en Fraijanes, hubo deslizamiento en las laderas del volcán Poás, 
daños en Alajuela, Heredia y San José y una secuela de 37 temblores (González Víquez: 1994, 234).

15. Aguaro. Liquore specie di grappa.

Guaro.

16. Il nome della posizione in cui giaceva il nostro accampamento alle falde d'una montagna lungo il Ferro-Carril, Vallata fonda.

El nombre del sitio donde se situaba el campamento, en un valle, a las faldas de una montaña a lo largo del ferrocarril.

17. Altissimo monte dal quale discendemmo per andare a Cario. Qui c'era la ferrovía che ci condusse a Limone nel cui porto ci attendeva il vapore francese "Lesseps" che ci portò a Marsiglia.

Es un monte muy alto, del cual descendíamos para ir a Cario. Aquí se encontraba el andén que nos conducía a Limón, en cuyo puerto nos esperaba el vapor francés "Lesseps" que nos transportó hacia Marsella.

\section{Bibliografía}

Bariatti, Rita (1980) "La mano de obra italiana en el ferrocarril al Atlántico. 1887-1888”. Heredia: Universidad Nacional, Escuela de Historia, 29-71

Bariatti, Rita (1987) La inmigración italiana en Costa Rica. 1821-1963. Heredia: Universidad Nacional, tesis de grado, 92-140.

Bariatti, Rita (2001) Italianos en Costa Rica 1502-1952. De Cristóbal Colón a San Vito de Java. San José, Costa Rica: Universidad Autónoma de Centroamérica (UACA), primera edición.

Bariatti, Rita (2011) Italianos en América Central. De Cristóbal Colón a la segunda posguerra. San José, Costa Rica: Editorial Alma Mater, primera edición.

Disney, Dan (2011) "Is this how it's supposed to work?: Poetry as a radical technology in L2 Creative Writing classrooms". En International Journal for the Practice and Theory of Creative Writing, http://dx.doi.org/10.1080/14790726 .2011.573078

Fioravanti Bosi, Massimo (1904) Il canzoniere di un contadino, compilado por Avancinio Avancini. Milano, Italia: Ed. Vallardi.

Fioravanti Bosi, Massimo (1936) Poesie. A cura di Eravardo Fioravanti e Vittorio Parisi. Palmi Calabria, Italia: Edizioni Rassegna.

González Víquez, Cleto (1994). Temblores, terremotos, inundaciones y erupciones volcánicas en Costa Rica. 1608-1910. San José, Costa Rica: Editorial Tecnológica de Costa Rica, primera reimpresión.

Hall, Geoff (2003) "Poetry, Pleasure, and Second Language Learning Classrooms". En Applied Linguistics, volumen 24, número 3: 395-399, Oxford University Press.

Hanauer, David Ian (2001) "The Task of Poetry Reading and Second Language Learning”. En Applied Linguistics, volumen 22, número 3: 295-323, Oxford University Press.

Khatib, Mohammad et al. (2011) "Literature in EFL/ESL Classroom". En English Language Teaching, volumen 4, número 1: 201-208. Canadian Center of Science and Education. 
Lazar, Gillian (2005) Literature and Language Teaching: A Guide for Teachers and Trainers. Nueva York, Estados Unidos: Cambridge University Press, treceava edición (primera edición 1993).

Mattix, Micah (2002) "The Pleasure of Poetry Reading and Second Language Learning: A Response to David Hanauer". En Applied Linguistics, volumen 23, número 4: 515-518, Oxford University Press. 